\title{
Approximate Symmetries Analysis and Conservation Laws Corresponding to Perturbed Korteweg-de Vries Equation
}

\author{
Tahir Ayaz, ${ }^{1}$ Farhad Ali, ${ }^{1}$ Wali Khan Mashwani (D), ${ }^{1}$ Israr Ali Khan, ${ }^{1}$ Zabidin Salleh (D), \\ and Ikramullah ${ }^{3}$ \\ ${ }^{1}$ Institute of Numerical Sciences, Kohat University of Sciencee Technology, Kohat, Pakistan \\ ${ }^{2}$ Department of Mathematics, Faculty of Ocean Engineering Technology and Informatics, Universiti Malaysia Terengganu, \\ Kuala Nerus 21030, Terengganu, Malaysia \\ ${ }^{3}$ Department of Physics, Kohat University of Sciencee Technology, Kohat, Pakistan
}

Correspondence should be addressed to Wali Khan Mashwani; mashwanigr8@gmail.com

Received 25 April 2021; Accepted 10 June 2021; Published 22 June 2021

Academic Editor: Zakia Hammouch

Copyright (C) 2021 Tahir Ayaz et al. This is an open access article distributed under the Creative Commons Attribution License, which permits unrestricted use, distribution, and reproduction in any medium, provided the original work is properly cited.

The Korteweg-de Vries (KdV) equation is a weakly nonlinear third-order differential equation which models and governs the evolution of fixed wave structures. This paper presents the analysis of the approximate symmetries along with conservation laws corresponding to the perturbed $\mathrm{KdV}$ equation for different classes of the perturbed function. Partial Lagrange method is used to obtain the approximate symmetries and their corresponding conservation laws of the KdV equation. The purpose of this study is to find particular perturbation (function) for which the number of approximate symmetries of perturbed KdV equation is greater than the number of symmetries of $\mathrm{KdV}$ equation so that explore something hidden in the system.

\section{Introduction}

Differential equations (DEs) are ubiquitous in modeling an extensive class of physical phenomena involving variation with respect to one or more independent variables. Therefore, DEs are broadly divided into ordinary DEs (ODEs) and partial DEs (PDEs). In different sectors of science and technology, PDEs have played a significant role. PDEs have numerous applications in mathematics, physics, fluid dynamics, mechanics, and physical chemistry. Modeling of PDEs under special conditions and constraints is advantageous in different situations for an effective manipulation of the varying phenomenon. The majority of real-world problems are almost nonlinear in nature, having no analytical solutions. In order to solve nonlinear problems, various approximations and techniques are used to gain high accuracy. In this regard, the approximate symmetry methods play a significant role. We have used the method of approximate Lie symmetry $[1,2]$, for PDEs to deal with the dynamical system more accurately. In the 1980s, the method of approximate Lie symmetry was developed by Baikov et al.
$[3,4]$. In obtaining the approximate solutions to such perturbed PDEs, the approximate symmetry method is an effective one. The extension of Lie's theory was mainly the basic reason behind the development of approximate symmetry, which deals with the systems by introducing small perturbation [5]. Symmetry applications to physical problems play a pivotal role in the development of conservation laws $[6,7]$. The widely recognized $\mathrm{KdV}$ equation is a mathematical model for the depiction of weak nonlinear long wavelength waves in various branches of engineering and physics. It explains how waves evolve due to comparable effects of weak nonlinearity and dispersion. A perturbed nonlinear wave equation is a class of approximate symmetries which is computed using two newly developed methods. For both methods, the associated invariant solution with the approximate symmetries is constructed. By discussing the advantages and disadvantages of each method, the symmetries and solutions are compared. So, the Lie group technique in finding the exact solution of a differential equation has lost its importance. But an approximate Lie group technique has been implemented and used in 
various methods for obtaining additional related information of differential equation. Perturbation analysis is one of the techniques which is used particularly for nonlinear systems.

This study is framed in the following manner: Section 2 is devoted to the development of exact symmetries and exact conservation laws of the $\mathrm{KdV}$ equation. The method to handle the approximate part of the $\mathrm{KdV}$ equation is developed in Section 3. The method so developed is applied to tackle the approximate part of the KdV equation for different cases and their corresponding conservation laws in Section 4 . The work is concluded by describing the highlights in Section 5.

\section{Exact Symmetries and Conservation Laws of the Korteweg-de Vries (KdV) Equation}

The exact symmetries and conservation laws in the current study for the work considered in $[8,9]$ are worked out as follows:
The Korteweg-de Vries (KdV) equation which is a thirdorder nonlinear partial differential equation is

$$
\mu_{t}-6 \mu \mu_{x}+\mu_{x x x}=0 .
$$

The infinitesimal symmetry operator is

$$
\begin{aligned}
\mathbf{X}^{[3]}= & \phi \frac{\partial}{\partial x}+\varrho \frac{\partial}{\partial t}+\varphi \frac{\partial}{\partial \mu}+\varphi^{t} \frac{\partial}{\partial \mu_{t}}+\varphi^{x} \frac{\partial}{\partial \mu_{x}}+\varphi^{x x} \frac{\partial}{\partial \mu_{x x}} \\
& +\varphi^{x t} \frac{\partial}{\partial \mu_{x t}}+\varphi^{t t} \frac{\partial}{\partial \mu_{t t}}+\varphi^{x x x} \frac{\partial}{\partial \mu_{x x x}} .
\end{aligned}
$$

Applying this symmetry operator on (1),

$$
\mathbf{X}^{[3]}\left(\mu_{t}-6 \mu \mu_{x}+\mu_{x x x}\right)=0,
$$

we get

$$
\varphi^{t}-6 \mu \varphi^{x}-6 \varphi \mu_{x}+\varphi^{x x x}=0 .
$$

The expanded form of equation (4) is

$$
\begin{aligned}
& {\left[\varphi_{t}-\phi_{t} \mu_{x}+\left(\varphi_{\mu}-\varrho_{t}\right) \mu_{t}-\phi_{\mu} \mu_{x} \mu_{t}-\varrho \mu \mu_{t}^{2}\right]-6 \mu\left[\varphi_{x}+\left(\varphi_{\mu}-\phi_{x}\right) \mu_{x}-\varrho_{x} \mu_{t}-\phi_{\mu} \mu_{x}^{2}-\varrho_{x} \mu_{x} \mu_{t}\right]} \\
& -6 \varphi \mu_{x}+\left[\varphi x x x+\left(3 \varphi_{\mu x x}-\phi_{x x x}\right) \mu_{x}-\varrho_{x x x} \mu_{t}+\left(3 \varphi_{\mu \mu x}-3 \phi_{x x \mu}\right)\right. \\
& \mu_{x}^{2}-3 \varrho_{\mu x x} \mu_{x} \mu_{t}+\left(3 \varphi_{\mu x}-3 \phi_{x x}\right) \mu_{x x}-3 \varrho_{x x} \mu_{x t}+\left(\varphi_{\mu \mu \mu}-\phi_{\mu \mu x}\right) \mu_{x}^{3} \\
& -3 \varrho_{\mu \mu x} \mu_{x}^{2} \mu_{t}+\left(3 \varphi_{\mu \mu}-9 \phi_{\mu x}\right) \mu_{x} \mu_{x x}-6 \varrho_{x \mu} \mu_{x} \mu_{x t}-3 \varrho_{\mu x} \mu_{t} \mu_{x x}+\left(\varphi_{\mu}-3 \phi_{x}\right) \mu_{x x x} \\
& -3 \varrho_{x} \mu_{x x t}-\phi_{\mu \mu \mu} \mu_{x}^{4}-\varrho_{\mu \mu \mu} \mu_{x}^{3} \mu_{t}-6 \phi_{\mu \mu} \mu_{x}^{2} \mu_{x x}-3 \varrho_{\mu \mu} \mu_{x}^{2} \mu_{x t}-3 \varrho_{\mu \mu} \mu_{x} \mu_{t} \mu_{x x} \\
& -4 \varphi_{\mu} \mu_{x} \mu_{x x x}-3 \varrho_{\mu} \mu_{x} \mu_{x x t}-3 \phi_{\mu} \mu_{x x}^{2}-3 \varrho_{\mu} \mu_{x x} \mu_{x t}-\varrho_{\mu} \mu_{t} \mu_{x x x}=0 .
\end{aligned}
$$

Substituting equation (1) in equation (5), we get

$$
\begin{aligned}
& \varphi_{t}-\phi_{t} \mu_{x}+\left(\varphi_{\mu}-\varrho_{t}\right) \mu_{t}-\phi_{\mu} \mu_{x} \mu_{t}-\varrho_{\mu} \mu_{t}^{2}-6 \varphi \mu_{x}-6 \mu\left[\varphi_{x}+\left(\varphi_{\mu}-\phi_{x}\right) \mu_{x}-\varrho_{x} \mu_{t}-\phi_{\mu} \mu_{x}^{2}-\varrho_{\mu} \mu_{x} \mu_{t}\right] \\
& +\varphi_{x x x}+\left(3 \varphi_{x x \mu}-\phi_{x x x}\right) \mu_{x}-\varrho_{x x x} \mu_{t}+\left(3 \varphi_{x \mu \mu}-3 \phi_{x x \mu}\right) \mu_{x}^{2} \\
& -3 \varrho_{x x \mu} \mu_{x} \mu_{t}+\left(3 \varphi_{x \mu}-3 \phi_{x x}\right) \mu_{x x}-3 \varrho_{x x} \mu_{x t}+\left(\varphi_{\mu \mu \mu}-\phi_{x \mu \mu}\right) \mu_{x}^{3}-3 \varrho_{x \mu \mu} \mu_{x}^{2} \mu_{t} \\
& +\left(3 \varphi_{\mu \mu}-9 \phi_{x \mu}\right) \mu_{x} \mu_{x x}-6 \varrho_{x \mu} \mu_{x} \mu_{x t}-3 \varrho_{x \mu} \mu_{t} \mu_{x x}+6 \mu\left(\varphi_{\mu}-3 \phi_{x}\right) \mu_{x}-\left(\varphi_{\mu}-3 \phi_{x}\right) \mu_{t} \\
& -3 \varrho_{x} \mu_{x x t}-\phi_{\mu \mu \mu} \mu_{x}^{4}-\varrho_{\mu \mu \mu} \mu_{x}^{3} \mu_{t}-6 \phi_{\mu \mu} \mu_{x}^{2} \mu_{x x}-3 \varrho_{\mu \mu} \mu_{x}^{2} \mu_{x t}-3 \varrho_{\mu \mu} \mu_{x} \mu_{t} \mu_{x x} \\
& -24 \mu \phi_{\mu} \mu_{x}^{2}+4 \phi_{\mu} \mu_{x} \mu_{t}-3 \varrho_{\mu} \mu_{x} \mu_{x x t}-3 \phi_{\mu} \mu_{x x}^{2}-3 \varrho_{\mu} \mu_{x x} \mu_{x t}-6 \mu \varrho_{\mu} \mu_{x} \mu_{x t}+\varrho_{\mu} \mu_{t}^{2}=0 .
\end{aligned}
$$


Comparing the coefficients of various terms, we get the coefficients and monomials, as shown in Table 1.

Table 1 yields the required set of PDEs as follows:

$$
\begin{aligned}
\varrho_{\mu} & =0, \\
\phi_{\mu} & =0, \\
\varrho_{x} & =0, \\
3 \phi_{x}-\varrho_{t} & =0 .
\end{aligned}
$$

Form (10),

$$
\begin{aligned}
\phi_{x x} & =0 \\
\Rightarrow \varphi_{x x \mu} & =\phi_{x x x} \\
\Rightarrow \varphi_{x x \mu} & =0, \\
\varphi & =\frac{-1}{6} \phi_{t}-2 \mu \phi_{x} .
\end{aligned}
$$

As

$$
\phi_{x}=\frac{1}{3} \varrho_{t}
$$

therefore,

$$
\begin{aligned}
\varphi & =-\frac{1}{6} \phi_{t}-\frac{2}{3} \mu \varrho_{t}, \\
\varphi_{t} & =-\frac{1}{6} \phi_{t t}-\frac{2}{3} \mu \varrho_{t t}, \\
\varphi_{x} & =-\frac{1}{6} \phi_{x t}-\frac{2}{3} \mu \varrho_{t x}, \\
\varphi_{x x x} & =0, \\
\phi_{t t} & =0, \\
\varrho_{t t} & =0 .
\end{aligned}
$$

Let

$$
\begin{aligned}
\varrho & =A(t) \\
\Rightarrow A_{t t}(t) & =0 .
\end{aligned}
$$

Integrating twice with respect to $t$ yields

$$
\begin{aligned}
\Rightarrow A_{t}(t) & =k_{1} \\
\Rightarrow A(t) & =k_{1} t+k_{2} \\
\Rightarrow \varrho & =k_{1} t+k_{2} .
\end{aligned}
$$

From (10),

$$
\begin{aligned}
3 \phi_{x}-\varrho_{t} & =0, \\
\phi_{\mu} & =0 \Rightarrow \phi=B(x) t, \\
\phi_{x} & =\frac{1}{3} \varrho_{t} \\
\Rightarrow \phi_{x} & =\frac{1}{3} k_{1} .
\end{aligned}
$$

Integrating with respect to " $x$,"

$$
\begin{gathered}
\Rightarrow \phi=\frac{1}{3} k_{1} x+D(t) \\
\Rightarrow \phi_{t t}=D_{t t}(t)=0 \\
\Rightarrow D(t)=k_{3} t+k_{4} \\
\Rightarrow \phi=\frac{1}{3} k_{1} x+k_{3} t+k_{4} .
\end{gathered}
$$

From (13),

$$
\begin{aligned}
\varphi & =-\frac{1}{6} \phi_{t}-\frac{2}{3} \mu \varrho_{t} \\
& =-\frac{1}{6} k_{3}-\frac{2}{3} k_{1} \mu .
\end{aligned}
$$

The general solution is

$$
\begin{aligned}
& \varphi=-\frac{2}{3} k_{1} \mu-\frac{1}{6} k_{3}, \\
& \varrho=k_{1} t+k_{2}, \\
& \phi=\frac{1}{3} k_{1} x+k_{3} t+k_{4} .
\end{aligned}
$$

Hence, the Lie symmetry generators for the $\mathrm{KdV}$ equation are given, as shown in Table 2.

\section{A New Procedure to Find the Approximate Symmetries}

This section explains the development of the method for the approximate symmetries of the KdV equation. The $\mathrm{KdV}(1)$ is perturbed with the function $f(x, t, \mu(x, t), \mu(t, x))$ as

$$
\mu_{t}-6 \mu \mu_{x}+\mu_{x x x}+\varepsilon f(x, t, \mu(x, t), \mu(t, x))=0,
$$

where $\varepsilon$ is a small parameter, causing the required perturbation in the $\mathrm{KdV}$ equation. The exact and approximate parts of (22) are

$$
\begin{aligned}
& E_{e}=\mu_{t}-6 \mu \mu_{x}+\mu_{x x x}, \\
& E_{a}=f(x, t, \mu(x, t)) .
\end{aligned}
$$


TABle 1: The exact symmetries of the given partial differential equation (PDE).

\begin{tabular}{lcc}
\hline Coefficients & Monomials \\
\hline$\varphi_{t}-6 \mu \varphi_{x}+\varphi_{x x x}=0$ & 1 \\
$-\phi_{t}-6 \varphi-6 \mu\left(\varphi_{\mu}-\phi_{x}\right)+3 \varphi_{x x \mu}-\phi_{x x x}+6 \mu\left(\varphi_{\mu}-3 \phi_{x}\right)=0$ & $\mu_{x}$ \\
$-\phi_{\mu}+6 \mu \varrho_{\mu}-3 \varrho_{x x \mu}+4 \phi_{\mu}=0$ & $\mu_{x} \mu_{t}$ \\
$\varphi_{\mu}-\varrho_{t}+6 \mu \varrho_{x}-\varrho_{x x x}-\left(\varphi_{\mu}-3 \phi_{x}\right)=0$ & $\mu_{t}$ \\
$-\varrho_{\mu}+\varrho_{\mu}=0$ & $\mu_{t}^{2}$ \\
$6 \mu \phi_{\mu}+3 \varphi_{x \mu \mu}-3 \phi_{x x \mu}-24 \mu \phi_{\mu}=0$ & $\mu_{x}^{2}$ \\
$3 \varphi_{x \mu}-3 \phi_{x x}=0$ & $\mu_{x x}$ \\
$-3 \varrho_{x x}=0$ & $\mu_{x t}$ \\
$\varphi_{\mu \mu \mu}-\phi_{x \mu \mu}=0$ & $\mu_{x}^{3}$ \\
$-3 \varrho_{x \mu \mu}=0$ & $\mu_{x}^{2} \mu_{t}$ \\
$3 \varphi_{\mu \mu}-9 \phi_{x \mu}=0$ & $\mu_{x} \mu_{x x}$ \\
$-6 \varrho_{x \mu}-6 \mu \varrho_{\mu}=0$ & $\mu_{x} \mu_{x t}$ \\
$-3 \varrho_{x \mu}=0$ & $\mu_{t} \mu_{x x}$ \\
$-3 \varrho_{x}=0$ & $\mu_{x x t}$ \\
$\phi_{\mu \mu \mu}=0$ & $\mu_{x}^{4}$ \\
$\varrho_{\mu \mu \mu}=0$ & $\mu_{x}^{3} \mu_{t}$ \\
$\phi_{\mu \mu}=0$ & $\mu_{x}^{2} \mu_{x x}$ \\
$\varrho_{\mu \mu}=0$ & $\mu_{x}^{2} \mu_{x t}$ \\
$\varrho_{\mu \mu}=0$ & $\mu_{x} \mu_{t} \mu_{x x}$ \\
$\varrho_{\mu}=0$ & $\mu_{x} \mu_{x x t}$ \\
$\phi_{\mu}=0$ & $\mu_{x x}^{2}$ \\
$\varrho_{\mu}=0$ & $\mu_{x x} \mu_{x t}$ \\
\hline
\end{tabular}

TABLE 2: Lie symmetry generator of KdV equation.

Lie symmetry generators

$X_{1}=(1 / 3) x(\partial / \partial x)+t(\partial / \partial t)-(2 / 3) \mu(\partial / \partial \mu)$

$X_{2}=(\partial / \partial t)$

$X_{3}=(\partial / \partial)$

$X_{4}=(\partial / \partial x)$

Equation (22) can now be written in a more compact form as

$$
E_{e}+\varepsilon E_{a}=0
$$

On similar footing, we can combine the exact and approximate Lie symmetries as

$$
\mathbf{X}=\mathbf{X}_{e}+\varepsilon \mathbf{X}_{a} .
$$

Here,

$$
\mathbf{X}_{e}=\phi_{e} \frac{\partial}{\partial x}+\varrho_{e} \frac{\partial}{\partial t}+\varphi_{e} \frac{\partial}{\partial \mu}
$$

is the exact Lie symmetry generator, and

$$
\mathbf{X}_{a}=\phi_{a} \frac{\partial}{\partial x}+\varrho_{a} \frac{\partial}{\partial t}+\varphi_{a} \frac{\partial}{\partial \mu}
$$

is the approximate Lie symmetry generator. Furthermore, $\phi$, $\varrho$, and $\varphi$ are the unknown functions of $x, t$, and $\mu$, respectively.

Now, applying the generator $\mathbf{X}$ on (24), we have

$$
\left(\mathbf{X}_{e}+\varepsilon \mathbf{X}_{a}\right)\left(E_{e}+\varepsilon E_{a}\right)=0
$$

which yields

$$
\mathbf{X}_{e} E_{e}+\varepsilon\left(\mathbf{X}_{a} E_{e}+\mathbf{X}_{e} E_{a}\right)+O\left(\varepsilon^{2}\right)=0 .
$$

The comparison of coefficients of $\varepsilon^{0}$ and $\varepsilon^{1}$, respectively, yields the exact and approximate symmetries of the corresponding PDEs as in the following:

$$
\begin{aligned}
\mathbf{X}_{e} E_{e} & =0, \\
\mathbf{X}_{a} E_{e}+\mathbf{X}_{e} E_{a} & =0 .
\end{aligned}
$$

The latter equation additionally gives the approximate Lie symmetries, which will not only provide the approximate conservation laws involved in the dynamics of the $\mathrm{KdV}$ equation but will also give the unknown function $f\left(x, t, \mu(x, t), \mu_{i}(t, x)\right)[8,10]$.

\section{Approximate Symmetries and Corresponding Conservation Laws of the KdV Equation}

In this section, we apply the developed method to find out the approximate symmetries. This method is applied and discussed for different cases. Considering the perturbed $\mathrm{KdV}$ equation $[6,11,12]$,

$$
\mu_{t}-6 \mu \mu_{x}+\mu_{x x x}+\varepsilon f\left(x, y, n, t, n_{t}, n_{x}, m_{t}, m_{x}\right)=0 .
$$

By employing the method developed in [13-15] for the expansion of $\mu$,

$$
\mu=m+\varepsilon n .
$$

Using this expansion in (31), 


$$
\begin{aligned}
\left(m_{t}+\varepsilon n_{t}\right)-6(m+\varepsilon n)\left(m_{x}+\varepsilon n_{x}\right)+\left(m_{x x x}+\varepsilon n_{x x x}\right) & =\varepsilon f\left(x, y, n, t, n_{t}, n_{x}, m_{t}, m_{x}\right), \\
m_{t}+\varepsilon n_{t}-6 m m_{x}-6 \varepsilon m n_{x}-6 \varepsilon n m_{x}-6 \varepsilon^{2} n n_{x}+m_{x x x}+\varepsilon n_{x x x} & =\varepsilon f\left(x, y, n, t, n_{t}, n_{x}, m_{t}, m_{x}\right), \\
\left(m_{t}-6 m m_{x}+m_{x x x}\right)+\varepsilon\left(n_{t}-6 m n_{x}-6 n m_{x}+n_{x x x}\right)+\varepsilon^{2}\left(-6 n n_{x}\right) & =\varepsilon f\left(x, y, n, t, n_{t}, n_{x}, m_{t}, m_{x}\right) .
\end{aligned}
$$

Equation (33) in more compact form is (neglecting higher power of $\epsilon$ )

$$
\Delta_{e}+\varepsilon \Delta_{a}=0
$$

The comparison of the coefficients of $\epsilon^{0}$ and $\epsilon^{1}$ in (33) gives

$$
\begin{aligned}
\Delta_{e}:=m_{t}-6 m m_{x}+m_{x x x} & =0 \\
\Delta_{a}:=n_{t}-6 m n_{x}-6 n m_{x}+n_{x x x}-f\left(x, y, n, t, n_{t}, n_{x}, m_{t}, m_{x}\right) & =0 .
\end{aligned}
$$

The Lie symmetry generator is

$$
\varrho_{t t}=0 \text {, }
$$

$$
\mathbf{X}=\mathbf{X}_{e}+\varepsilon \mathbf{X}_{a}=0
$$

Here,

$$
\begin{aligned}
& \mathbf{X}_{e}=\phi_{e} \frac{\partial}{\partial x}+\varrho_{e} \frac{\partial}{\partial t}+\varphi_{e} \frac{\partial}{\partial m}+\phi_{e} \frac{\partial}{\partial n} \\
& \mathbf{X}_{a}=\phi_{a} \frac{\partial}{\partial x}+\varrho_{a} \frac{\partial}{\partial t}+\varphi_{a} \frac{\partial}{\partial m}+\phi_{a} \frac{\partial}{\partial n}
\end{aligned}
$$

Applying the Lie generator,

$$
\begin{aligned}
\mathbf{X}\left(\Delta_{e}+\varepsilon \Delta_{a}\right) & =0 \\
\left(\mathbf{X}_{e}+\varepsilon \mathbf{X}_{a}\right)\left(\Delta_{e}+\varepsilon \Delta_{a}\right) & =0
\end{aligned}
$$

which gives us

$$
\begin{aligned}
\mathbf{X}_{e} \Delta_{e}+\varepsilon\left(\mathbf{X}_{a} \Delta_{e}+\mathbf{X}_{e} \Delta_{a}\right)+o\left(\varepsilon^{2}\right) & =0 \\
\mathbf{X}_{e} \Delta_{e} & =0 \\
\mathbf{X}_{a} \Delta_{e}+\mathbf{X}_{e} \Delta_{a} & =0
\end{aligned}
$$

As

We now discuss the following cases in a bit detail.

Case I. Let

$$
f\left(x, y, n, t, n_{t}, n_{x}, m_{t}, m_{x}\right)=-m_{t}-n_{t} .
$$

Then, determining the system of PDEs from (35),

$$
\varrho_{m}=0
$$$$
\phi_{t}=0 \text {, }
$$$$
\varrho_{n}=0 \text {, }
$$$$
\phi_{n}=0 \text {, }
$$$$
\phi_{m}=0,
$$$$
\varrho_{x}=0 \text {, }
$$$$
\phi_{x}=\frac{3}{\varrho_{t}},
$$

$$
\begin{aligned}
& \varphi=\frac{-2}{3} m \varrho_{t}, \\
& \phi=-\frac{2}{3} \varrho_{t} n .
\end{aligned}
$$

$$
\begin{gathered}
\frac{\partial \varrho}{\partial m}=0, \\
\frac{\partial \varrho}{\partial n}=0, \\
\frac{\partial \varrho}{\partial x}=0,
\end{gathered}
$$


which implies that " $\varrho$ " is the function of " $t$ " alone. Therefore,

$$
\varrho_{t t}=0 \text {. }
$$

Integrating the above equation twice with respect to " $t$ " yields

$$
\varrho=c_{1} t+c_{2} \text {. }
$$

Also,

$$
\begin{gathered}
\frac{\partial \phi}{\partial t}=0, \\
\frac{\partial \phi}{\partial m}=0, \\
\frac{\partial \phi}{\partial n}=0,
\end{gathered}
$$

which shows that " $\phi$ " is the function of " $x$ " alone. Therefore,

$$
\frac{\partial \phi}{\partial x}=\frac{1}{3} \varrho t
$$

Putting the value of " $\varrho_{t}$ " in (46), we get

$$
\frac{\partial \phi}{\partial x}=\frac{1}{3} c_{1} \text {. }
$$

Integrating (47), we get

$$
\phi=\frac{1}{3} c_{1} x+c_{3} .
$$

Now,

$$
\varphi=-\frac{2}{3} m \varrho_{t} .
$$

Putting the value of " $\varrho_{t}$ ” in (49),

$$
\varphi=-\frac{2}{3} m c_{1} .
$$

By taking

$$
\phi=-\frac{2}{3} \varrho_{t} n,
$$

and putting the value of " $\varrho_{t}$ " in (51),

$$
\phi=-\frac{2}{3} c_{1} n .
$$

Therefore,

$$
\begin{aligned}
& \phi=\frac{1}{3} c_{1} x+c_{3}, \\
& \varrho=c_{1} t+c_{2}, \\
& \varphi=\frac{-2}{3} m c_{1}, \\
& \phi=\frac{-2}{3} c_{1} n .
\end{aligned}
$$

The corresponding symmetry generators are tabulated in Table 3.

4.1. Conservation Laws. The conservation laws are developed as in the following:

$$
\mathbf{X}_{1}\left(\psi\left(x, y, n, t, n_{t}, n_{x}, m_{t}, m_{x}\right)\right)=0
$$

$$
\begin{gathered}
\left(\frac{1}{3} x \frac{\partial}{\partial x}+t \frac{\partial}{\partial t}-\frac{2}{3} \frac{\partial}{\partial n}-\frac{2}{3} m \frac{\partial}{\partial m}\right) \psi=0 \\
\frac{1}{3} x \psi_{x}+t \psi_{t}-\frac{2}{3} \psi_{n}-\frac{2}{3} m \psi_{m}=0 \\
3 \frac{d x}{x}=\frac{d t}{t}=\frac{d n}{(-2 / 3)}=\frac{-3}{2} \frac{d m}{m}=\frac{d \psi}{0} .
\end{gathered}
$$

Now, by taking

$$
\begin{aligned}
3 \frac{d x}{x} & =\frac{d t}{t} \Rightarrow x^{3}=c_{1} t \Rightarrow c_{1}=\frac{x^{3}}{t}, \\
3 \frac{d x}{x} & =\frac{d n}{(-2 / 3)} \Rightarrow \ln x^{3}=-\frac{2}{3} n+c_{2} \Rightarrow c_{2}=x^{3} e^{(3 / 2)} n, \\
3 \frac{d x}{x} & =\frac{-3}{2} \frac{d m}{m} \Rightarrow x^{3}=c_{3} m^{-} \frac{3}{2} \Rightarrow c_{3}=x^{3} m^{(3 / 2)}, \\
\frac{d t}{t} & =\frac{d n}{(-2 / 3)} \Rightarrow \ln t=-\frac{2}{3} n+c_{4} \Rightarrow c_{4}=t e^{(3 / 2)} n, \\
\frac{d t}{t} & =\frac{-3}{2} \frac{d m}{m} \Rightarrow t=c_{5} m^{-} \frac{3}{2} \Rightarrow c_{5}=t m \frac{3}{2}, \\
\frac{d n}{(-2 / 3)} & =\frac{-3}{2} \frac{d m}{m} \Rightarrow n+c_{6}=\ln m \Rightarrow c_{6}=m e^{-n},
\end{aligned}
$$

so

$$
\begin{aligned}
\psi= & c_{1}+c_{2}+c_{3}=\frac{x^{3}}{t}+x^{3} e^{(3 / 2)} n+x^{3} m^{(3 / 2)} \\
& +t e^{(3 / 2)} n+t m \frac{3}{2}+m e^{-n} .
\end{aligned}
$$

Furthermore,

$$
\begin{aligned}
X_{2}\left(\psi\left(x, y, n, t, n_{t}, n_{x}, m_{t}, m_{x}\right)\right) & =0, \\
\psi_{t} & =0 \Rightarrow \psi=c, \\
X_{3}\left(\psi\left(x, y, n, t, n_{t}, n_{x}, m_{t}, m_{x}\right)\right) & =0, \\
\psi_{x} & =0 \Rightarrow \psi=c .
\end{aligned}
$$

Following are the symmetries and their corresponding conservation laws of Case 1 .

Case 2. Let

$$
f\left(x, y, n, t, n_{t}, n_{x}, m_{t}, m_{x}\right)=-m_{x} .
$$


TABLE 3: Lie symmetry generators.

Lie symmetry generators

$\mathbf{X}_{1}=(1 / 3) x(\partial / \partial x)+t(\partial / \partial t)-(2 / 3)(\partial / \partial n)-(2 / 3) m(\partial / \partial m)$

$\mathbf{X}_{2}=(\partial / \partial t)$

$\mathbf{X}_{3}=(\partial / \partial x)$

From (35), we get after comparing the coefficients of $\epsilon^{0}$ and $\epsilon^{1}$,

$$
\begin{aligned}
m_{t}-6 m m_{x}+m_{x x x} & =0, \\
n_{t}-6 m n_{x}-6 n m_{x}+n_{x x x}-m_{x} & =0 .
\end{aligned}
$$

Applying (36) to (59) yields the following system of PDEs:

$$
\begin{aligned}
\phi_{t} & =0, \\
\phi_{n} & =0, \\
\phi_{m} & =0, \\
\phi_{m} & =0, \\
\phi_{n} & =\frac{6 \phi}{n+1}, \\
\phi_{x} & =\frac{1}{3} \varrho_{t}, \\
\phi_{x} & =0, \\
\varrho_{m} & =0, \\
\phi_{t t} & =0, \\
\varrho_{n} & =0, \\
\varphi & =\frac{-2}{3} \varrho_{t} m-\frac{1}{6} \phi_{t} \varrho_{x}=0, \\
\varrho_{t t} & =0 .
\end{aligned}
$$

Solving the above system of PDEs, we get the following results:

$$
\begin{aligned}
& \varphi=\frac{-2}{3} c_{1} m-\frac{1}{6} c_{4}, \\
& \phi=6 c_{3} n+c_{3}, \\
& \varrho=c_{1} t+c_{2}, \\
& \phi=\frac{1}{3} c_{1} x+c_{4} t+c_{5} .
\end{aligned}
$$

The approximate symmetries and their corresponding conservation laws in this case are given in Table 4 .

Case 3. For this case, take

$$
f\left(x, y, n, t, n_{t}, n_{x}, m_{t}, m_{x}\right)=-n_{x} .
$$

From (35), we get after comparing the coefficients of $\epsilon^{0}$ and $\epsilon^{1}$,

$$
\begin{aligned}
m_{t}-6 m m_{x}+m_{x x x} & =0, \\
n_{t}-6 m n_{x}-6 n m_{x}+n_{x x x}-n_{x} & =0 .
\end{aligned}
$$

This results in the following equations:

$$
\begin{aligned}
\phi_{t} & =0, \\
\phi_{t t} & =0, \\
\phi_{m} & =0, \\
\phi_{x} & =0, \\
\phi_{n} & =\frac{\phi}{n} \\
\phi_{n} & =0, \\
\phi_{x} & =0, \\
\varrho_{t} & =0, \\
\phi_{m} & =0, \\
\varrho_{m} & =0, \\
\varphi & =\frac{-1}{6} \phi_{t} \varrho_{n}=0, \\
\varrho & =c_{4}, \\
\varrho_{x} & =0, \\
\varphi & =c_{1} t+c_{2} . \\
\varphi & =0
\end{aligned}
$$

Following are the symmetries and corresponding conservation laws of this Case 3.

Case 4. For this case, take

$$
f\left(x, y, n, t, n_{t}, n_{x}, m_{t}, m_{x}\right)=m n,
$$

then the system defined in (35) gives

$$
\begin{aligned}
m_{t}-6 m m_{x}+m_{x x x} & =0, \\
n_{t}-6 m n_{x}-6 n m_{x}+n_{x x x}+m n & =0 .
\end{aligned}
$$

Applying (36) to (66), we get the following set of PDEs: 
TABLE 4: Lie symmetry generators and corresponding conservation laws.

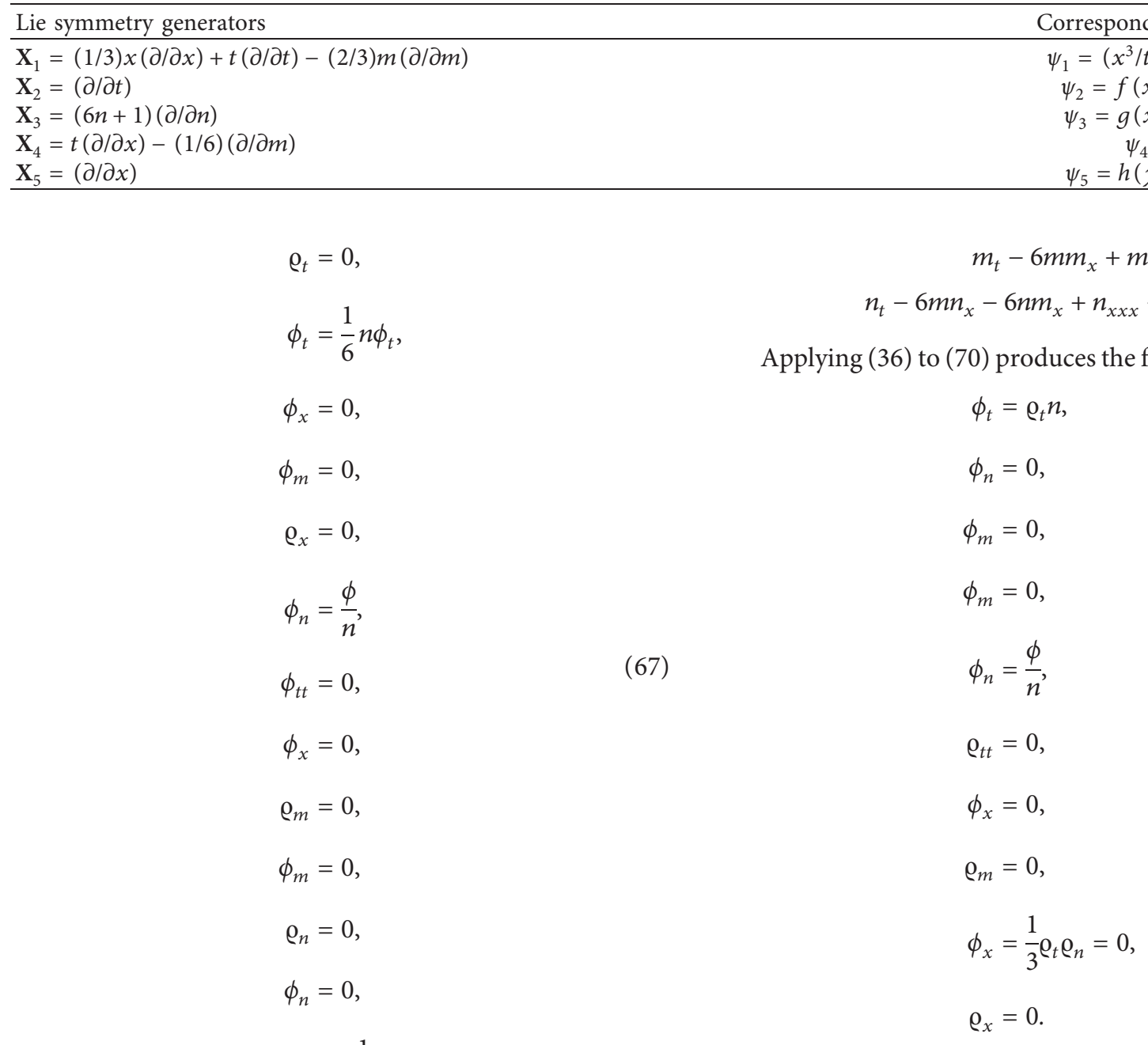

Solving the above equations, we get

The above equations yield

$$
\begin{aligned}
& \phi=\frac{c_{1} x}{3}+t c_{3}+c_{4}, \\
& \varrho=c_{1} t+c_{2}, \\
& \varphi=\frac{-2}{3} m \varrho_{t} c_{1}-\frac{1}{6} \phi_{t} c_{3}, \\
& \phi=n\left(c_{1} t+c_{5}\right) . \\
& \phi=c_{1} t+c_{2}, \\
& \varrho=c_{3} \text {, } \\
& \varphi=\frac{-1}{6} c_{1},
\end{aligned}
$$$$
\phi=\frac{1}{6} n\left(c_{1} t+6 c_{4}\right)
$$

Following are the symmetries and corresponding conservation laws of Case 4.

Case 5. Let

$$
f\left(x, y, n, t, n_{t}, n_{x}, m_{t}, m_{x}\right)=-n,
$$

then the system defined in (35) gives
Following are the symmetries and corresponding conservation laws of Case 5.

Case 6. Assume

$$
f\left(x, y, n, t, n_{t}, n_{x}, m_{t}, m_{x}\right)=-n m_{t},
$$

then the system defined in (35) gives

$$
\begin{aligned}
m_{t}-6 m m_{x}+m_{x x x} & =0, \\
n_{t}-6 m n_{x}-6 n m_{x}+n_{x x x}-n m_{t} & =0 .
\end{aligned}
$$

Applying (36) to (74) results in the following set of PDEs: 
TABLE 5: Lie symmetry generators and corresponding conservation laws.

\begin{tabular}{lc}
\hline Lie symmetry generators & Corresponding conservation laws \\
\hline $\mathbf{X}_{1}=(1 / 3) x(\partial / \partial x)+t(\partial / \partial t)+n t(\partial / \partial n)-(2 / 3) m(\partial / \partial m)$ & $\psi_{1}=\left(x^{3} / t\right)+x^{3}+x^{3} m^{(3 / 2)}+e^{t} n+t m^{(3 / 2)}$ \\
$\mathbf{X}_{2}=(\partial / \partial t)$ & $\psi_{2}=f\left(x, y, n, t, n_{t}, n_{x}, m_{t}, m_{x}\right)$ \\
$\mathbf{X}_{3}=(\partial / \partial x)-(1 / 6)(\partial / \partial m)$ & $\psi_{3}=x+6 m$ \\
$\mathbf{X}_{4}=(\partial / \partial x)$ & $\psi_{4}=g\left(y, n, t, n_{t}, n_{x}, m_{t}, m_{x}\right)$ \\
$\mathbf{X}_{5}=n(\partial / \partial n)$ & $\psi_{5}=h\left(x, y, t, n_{t}, n_{x}, m_{t}, m_{x}\right)$ \\
\hline
\end{tabular}

TABLE 6: Lie symmetry generator and corresponding conservation laws.

\begin{tabular}{lc}
\hline Lie symmetry generators & Corresponding conservation laws \\
\hline $\mathbf{X}_{1}=(1 / 3) x(\partial / \partial x)+t(\partial / \partial t)-(2 / 3)(\partial / \partial n)-(2 / 3) m(\partial / \partial m)$ & $\psi_{1}=\left(x^{3} / t\right)+x^{3} e^{(3 / 2)} n+x^{3} m^{(3 / 2)}+t e^{(3 / 2)} n+t m^{(3 / 2)}+m e^{-n}$ \\
$\mathbf{X}_{2}=(\partial / \partial t)$ & $\psi_{2}=f\left(x, y, n, n_{t}, n_{x}, m_{t}, m_{x}\right)$ \\
$\mathbf{X}_{3}=(\partial / \partial x)$ & $\psi_{3}=g\left(y, n, t, n_{t}, n_{x}, m_{t}, m_{x}\right)$ \\
\hline
\end{tabular}

TABLE 7: Lie symmetry generators and corresponding conservation laws.

\begin{tabular}{lc}
\hline Lie symmetry generators & Corresponding conservation laws \\
\hline $\mathbf{X}_{1}=(-1 / 6)(\partial / \partial x)+t(\partial / \partial t)$ & $\psi_{1}=\left(e^{-6 x} / t\right)$ \\
$\mathbf{X}_{2}=x(\partial / \partial x)$ & $\psi_{2}=f\left(y, n, t, n_{t}, n_{x}, m_{t}, m_{x}\right)$ \\
$\mathbf{X}_{3}=n(\partial / \partial n)$ & $\psi_{3}=g\left(x, y, t, n_{t}, n_{x}, m_{t}, m_{x}\right)$ \\
$\mathbf{X}_{4}=(\partial / \partial t)$ & $\psi_{4}=h\left(x, y, n, n_{t}, n_{x}, m_{t}, m_{x}\right)$ \\
\hline
\end{tabular}

TABLE 8: Lie symmetry generator and corresponding conservation laws.

\begin{tabular}{lr}
\hline Lie symmetry generators & Corresponding conservation laws \\
\hline $\mathbf{X}_{1}=t(\partial / \partial x)+(1 / 6) n t(\partial / \partial n)-(1 / 6)(\partial / \partial m)$ & $\psi_{1}=\left(e^{(x / t)} / n^{6}\right)+n e^{m}+(x / t)+6 m$ \\
$\mathbf{X}_{2}=(\partial / \partial x)$ & $\psi_{2}=f\left(y, n, t, n_{t}, n_{x}, m_{t}, m_{x}\right)$ \\
$\mathbf{X}_{3}=(\partial / \partial t)$ & $\psi_{3}=g\left(x, y, n, n_{t}, n_{x}, m_{t}, m_{x}\right)$ \\
$\mathbf{X}_{4}=n(\partial / \partial n)$ & $\psi_{4}=h\left(x, y, t, n_{t}, n_{x}, m_{t}, m_{x}\right)$ \\
\hline
\end{tabular}

TABLE 9: Lie symmetry generators and corresponding conservation laws.

\begin{tabular}{lc}
\hline Lie symmetry generators & Corresponding conservation laws \\
\hline $\mathbf{X}_{1}=n(\partial / \partial n)$ & $\psi=f\left(x, y, t, n_{t}, n_{x}, m_{t}, m_{x}\right)$ \\
$\mathbf{X}_{2}=(\partial / \partial t)$ & $\psi=g\left(x, y, n, n_{t}, n_{x}, m_{t}, m_{x}\right)$ \\
$\mathbf{X}_{3}=(\partial / \partial x)$ & $\psi_{3}=h\left(y, n, t, n_{t}, n_{x}, m_{t}, m_{x}\right)$ \\
\hline
\end{tabular}




$$
\begin{aligned}
& \varrho_{t x}=0, \\
& \phi_{t}=0, \\
& \phi_{x}=0, \\
& \phi_{m}=0, \\
& \phi_{n}=0, \\
& \phi_{n}=\frac{\phi}{n}, \\
& \phi_{m}=0, \\
& \varrho_{n}=0, \\
& \phi_{x}=0, \\
& \varrho_{t}=0, \\
& \phi_{t}=0, \\
& \varrho_{m}=0 .
\end{aligned}
$$

Solving the above set of equations, we get

$$
\begin{aligned}
& \phi=c_{3}, \\
& \varrho=c_{2}, \\
& \varphi=0, \\
& \phi=c_{1} n .
\end{aligned}
$$

Following are the symmetries and corresponding conservation laws of Case 6.

\section{Conclusion}

The $\mathrm{KdV}$ equation is a $3 \mathrm{rd}$ order nonlinear partial differential equation which is modeled for waves on the surface of shallow water. It admits four Lie symmetries given in $\mathrm{Ta}-$ ble 2 . In this paper, approximate symmetry techniques are used for finding some classes of the $\mathrm{KdV}$ equations that admit more symmetries as compared to the exact $\mathrm{KdV}$ equations. We perturbed the $\mathrm{KdV}$ equation by different particular functions and found the corresponding Lie symmetries. We found two important classes for the perturbed $\mathrm{KdV}$ equation that admits five Lie symmetries. The Lie symmetries along with their conservation laws are given in Tables 2, 4 and 5. In both the tables, we have an extra symmetry which corresponds to an extra conservation law. This extra conservation law is an extra information hidden in the system, the perturbation procedure explored it. Sometimes, the symmetry does not exist for the exact equation, but perturbation enables the equation to admit a symmetry. We saw this phenomenon in this research work by comparing Tables 2-6. Table 1 contains the determining PDEs which provide the set of Lie symmetries admitted by the given PDE. We have 4 Lie symmetries given in Table 2 for exact PDE, while Tables 3 and 6 contain only three Lie symmetries; in these cases, we lose one symmetry (one conservation law). Tables 7 and 8 consist of four Lie symmetries which means that all the conservation laws are recovered in these cases. Table 9 includes the lie symmetry generators and corresponding conservation laws.

\section{Data Availability}

There are no specific data used in the study of this article.

\section{Conflicts of Interest}

The authors declare that they have no conflicts of interest.

\section{References}

[1] H. Liu, J. Li, and L. Liu, "Lie symmetry analysis, optimal systems and exact solutions to the fifth-order KdV types of equations," Journal of Mathematical Analysis and Applications, vol. 368, no. 2, pp. 551-558, 2010.

[2] T. A. Abassy, M. A. El-Tawil, and H. K. Saleh, "The solution of $\mathrm{KdV}$ and MKdV equations using adomian pade approximation," International Journal of Nonlinear Sciences and Numerical Simulation, vol. 5, no. 4, pp. 327-340, 2004.

[3] V. Baikov, R. Gazizov, and N. Ibragimov, "Approximate symmetries," Matematicheskii Sbornik, vol. 136, no. 3, pp. 435-450, 1988.

[4] V. A. Baikov, R. K. Gazizov, and N. K. Ibragimov, "Perturbation methods in group analysis," Journal of Soviet Mathematics, vol. 55, no. 1, pp. 1450-1490, 1991.

[5] V. A. Baikov and N. H. Ibragimov, "Continuation of approximate transformation groups via multiple time scales method," Nonlinear Dynamics, vol. 22, no. 1, pp. 3-13, 2000.

[6] K. Parand, M. Dehghan, A. R. Rezaei, and S. M. Ghaderi, "An approximation algorithm for the solution of the nonlinear lane-emden type equations arising in astrophysics using hermite functions collocation method," Computer Physics Communications, vol. 181, no. 6, pp. 1096-1108, 2010.

[7] G. W. Bluman, A. F. Cheviakov, and S. C. Anco, Applications Of Symmetry Methods To Partial Differential Equations, Vol. 168, Springer, Berlin, Germany, 2010.

[8] X. R. Hu, S. Y. Lou, and Y. Chen, "“Explicit solutions from eigenfunction symmetry of the korteweg-de vries equation," Physical Review E, vol. 85, no. 5, Article ID 056607, 2012.

[9] A. R. Adem and C. M. Khalique, "Symmetry reductions, exact solutions and conservation laws of a new coupled KdV system," Communications in Nonlinear Science and Numerical Simulation, vol. 17, no. 9, pp. 3465-3475, 2012.

[10] N. Xiaohua and P. Zuliang, "A new approach to solve perturbed nonlinear evolution equations through lie-backlund symmetry method," Applied Mathematics-A Journal of Chinese Universities, vol. 21, no. 1, pp. 45-51, 2006.

[11] Z. Zhi-Yong, Y. Xue-Lin, and C. Yu-Fu, "A new method to obtain approximate symmetry of nonlinear evolution equation from perturbations," Chinese Physics B, vol. 18, no. 7, pp. 2629-2633, 2009.

[12] X. Jiao, R. Yao, and S. Y. Lou, “Approximate similarity reduction for singularly perturbed boussinesq equation via symmetry perturbation and direct method," Journal of Mathematical Physics, vol. 49, no. 9, Article ID 093505, 2008.

[13] A. H. Bokhari, A. H. Kara, and F. D. Zaman, "Invariant solutions of certain nonlinear evolution type equations with 
small parameters," Applied Mathematics and Computation, vol. 182, no. 2, pp. 1075-1082, 2006.

[14] D. D. Ganji and A. Sadighi, "Application of he's homotopyperturbation method to nonlinear coupled systems of reaction-diffusion equations," International Journal of Nonlinear Sciences and Numerical Simulation, vol. 7, no. 4, pp. 411-418, 2006.

[15] J. H. He, "Addendum: new interpretation of homotopy perturbation method," International Journal of Modern Physics B, vol. 20, no. 18, pp. 2561-2568, 2006. 